\title{
EL ROL DE LA COOPERACIÓN DE CHINA EN LA TRANSFORMACIÓN ESTRUCTURAL DEL SUR GLOBAL
}

\author{
Javier Alberto Vadell ${ }^{1}$ \\ Giuseppe Lo Brutto ${ }^{2}$ \\ Alexandre Cesar Leite ${ }^{3}$ \\ Eduardo Crivelli ${ }^{4}$
}

Resumen: En lo que va del siglo $\mathrm{XXI}$, el régimen internacional de cooperación y ayuda para el desarrollo ha visto el aumento de iniciativas ejecutadas por distintas potencias emergentes y países en desarrollo, centrándose cada vez más alrededor de China, cuya peculiar forma de cooperar parece guiar la transformación estructural del Sur global. Por eso, con fin de discutir estas cuestiones, el presente artículo analiza y debate la literatura que argumenta sobre el "modelo" de cooperación SurSur chino, explorando sus características y las peculiares relaciones de China con los países en desarrollo, ante la crisis del régimen cooperación tradicional.

Palabras clave: Cooperación Sur-Sur; potencias emergentes; China; Sur global, Nueva Economía Estructural.

\section{O PAPEL DA COOPERAÇÃO CHINA NA TRANSFORMAÇÃO ESTRUTURAL DO SUL GLOBAL}

Resumo: Até agora, no século XXI, o regime internacional de ajuda e cooperação ao desenvolvimento tem visto um aumento nas iniciativas realizadas por diferentes potências emergentes e países em desenvolvimento, concentrando-se cada vez mais na China, cuja forma peculiar de cooperação parece guiar a transformação estrutural do Sul global. Portanto, para discutir essas questões, este artigo analisa e debate a literatura que discute o "modelo" de cooperação Sul-Sul chinesa, explorando suas características e as peculiares relações entre a China e os países em desenvolvimento, em face da crise. do regime tradicional de cooperação.

Palavras-chave: Cooperação Sul-Sul; potências emergentes; China; Sul Global, Nova Economia Estrutural.

\section{THE ROLE OF CHINA'S COOPERATION IN THE STRUCTURAL TRANSFORMATION OF THE GLOBAL SOUTH}

Abstract: So far in the 21st century, the international development aid and cooperation regime has seen an increase in initiatives carried out by different emerging powers and developing countries, focusing more and more around China,

\footnotetext{
1 Pontifícia Universidade Católica de Minas Gerais, Relações Internacionais, Belo Horizonte, Brasil, javier.vadell@gmail.com, https://orcid.org/0000-0002-5398-6083.

2 Benemérita Universidad Autónoma de Puebla, Instituto de Ciencias Sociales y Humanidades Alfonso "Vélez Pliego", Puebla, México, giuseppe.lobrutto@correo.buap.mx, https://orcid.org/00000002-8126-5013.

3 Universidade Estadual de Paraíba, Programa de Pós-Graduação em Relações Internacionais, Universidade Federal da Paraíba, Programa de Pós-Graduação em Gestão Pública e Cooperação Internacional, Paraíba, Brasil, alexccleite@gmail.com, https://orcid.org/0000-0002-0209-2717.

4 Benemérita Universidad Autónoma de Puebla, Facultad de Derecho y Ciencias Sociales. Puebla, México, eduardo.crivelli@correo.buap.mx, https://orcid.org/0000-0002-1825-0097.
} 
whose peculiar way of cooperating seems to guide the structural transformation of the global South. Therefore, in order to discuss these issues, this article analyzes and debates the literature that argues about the "model" of Chinese South-South cooperation, exploring its characteristics and the peculiar relations between China and developing countries, in the face of the crisis. of the traditional cooperation regime.

Keywords: South-South Cooperation; emerging powers; China; Global South, New Structural Economics.

\section{Introducción}

En lo que va del siglo XXI, la cooperación internacional para el desarrollo ha visto el aumento de iniciativas ejecutadas por países en desarrollo, lo cual se explica por la percepción del impacto diferencial de sus prácticas enfocadas en la consolidación del desarrollo internacional (MAWDSLEY, 2012). En este ámbito, China se ha caracterizado por ser un actor con una posición ambivalente, porque si bien los representantes chinos también se incluyen en el "Sur", como beneficiarios de ayuda, también quieren ser considerados iguales a los países "del Norte" (BERGAMASCHI y DURÁN, 2017).

En este sentido, Shaw, Cooper y Antkiewicz (2007) señalaron que "el nuevo siglo se ve afectado por la cambiante postura económica de las potencias emergentes y su creciente influencia tanto en la economía mundial como en la arquitectura institucional global" (SHAW, COOPER, y ANTKIEWICZ, 2007, p. 1260) y, un aspecto de estas transformaciones son los cambios en las prácticas y procedimientos para brindar asistencia a los países en desarrollo. En este sentido, surge la pregunta sobre ¿cuál es el papel de China frente a estos cambios en las prácticas y procedimientos en la prestación de asistencia a los países en desarrollo? A pesar del enorme crecimiento en las últimas décadas, China aún enfrenta muchos problemas internos en lo que concierne a desarrollo (LEITE, 2018; LIMA, 2019), que lo confirman en el "grupo de países del Sur Global"5. Por esa razón, se observa en los discursos oficiales de China el auto reconocimiento como un país en desarrollo y, por lo tanto, un actor de la cooperación Sur-Sur (CSS) (CHINA-SCPRC, 2014).

Basado en un enfoque conceptual de Economía Política Internacional, este artículo aborda las transformaciones actuales de la CSS y el papel de China en este proceso. La pregunta principal de este artículo es ¿qué tipo de CSS está

\footnotetext{
${ }^{5}$ Siguiendo a Polanyi-Levitt (2018), en este artículo se comprende al Sur Global como aquella región del planeta delineada por la explotación, las conquistas y el comercio de los centros de la economía mundo capitalista desde el siglo XVI y que en términos actuales refiere al conjunto de países en vías de desarrollo.
} 
impulsando, promoviendo y expandiendo China a través de sus ambiciosos proyectos de inversión e iniciativas comerciales de préstamos y ayuda? Así, la hipótesis que guía este trabajo es que la CSS de China retoma los postulados de la Conferencia de Bandung adaptándolos a los tiempos actuales, para una transformación estructural del sistema mundial capitalista que se ido centrando cada vez más entorno a este gigante asiático. En este escenario, el poder relacional entre China y el Sur Global (VADELL, RAMOS y NEVES, 2014), sobre todo en el campo económico de la tríada comercio, inversiones y ayuda, parece estar consolidando un nuevo tipo de "consenso". Este proceso liderado por China está modificando de manera estructural el régimen internacional de CSS desafiando el consenso ideológico existente sobre las prácticas económicas del neoliberalismo global.

Por lo tanto, este artículo tiene como objetivo analizar y debatir la literatura que argumenta sobre un "modelo" de CSS chino y explorar las características peculiares de las relaciones de China con los países en desarrollo, ante la crisis del régimen de cooperación tradicional ${ }^{6}$. El artículo comienza con una sección que abre la perspectiva teórica sobre los donantes emergentes, como nuevos actores en el sistema internacional; en la segunda parte, se analiza el nuevo papel de la CSS frente al sistema tradicional de cooperación occidental; el tercer apartado, aborda una discusión sobre la evolución de la CSS a lo largo del tiempo; y, finalmente, la cuarta sección, presenta algunas consideraciones finales que abordan la eficacia de la ayuda y un análisis de la propuesta china de "transformación estructural" (LIN y WANG, 2017a, 2017b), con el fin de comprender su despliegue en el Sur Global como un enfoque ampliado del desarrollo y los consiguientes cambios a nivel de principios, normas y procedimientos recreando la posibilidad de una nueva red consensuada de poder.

\footnotetext{
${ }^{6}$ La crisis contemporánea del régimen de ayuda o cooperación se comprende desde distintas dimensiones ya sea como "crisis identitaria" (LO BRUTTO 2017; DOMÍNGUEZ, 2016, 2011; LO BRUTTO y GONZÁLEZ, 2016) o una "crisis existencial" (GORE, 2013, ESTEVES y ASSUNÇÃO, 2014). En todo caso, se podría decir que el mundo presencia una transformación global del actual régimen de cooperación internacional a través de la expansión global de China apalancada en su fortaleza económica. El enfoque y la fuerza de la CSS china está ganando protagonismo debido al desafío que supone competir con el modelo de Cooperación y ayuda de la OCDE, apareciendo como una opción más atractiva para los países del Sur Global.
} 


\section{La cooperación de las potencias medias y emergentes}

La distinción entre potencias medias tradicionales y emergentes ${ }^{7}$ es una característica compartida entre los debates del siglo XXI de las Relaciones Internacionales y sobre todo de los de la Economía Política Internacional ${ }^{8}$ (NOLTE, 2007, HUELSZ, 2009). Para Lima (2013), una potencia media se califica por su carácter multifacético de comportamiento en el ámbito internacional, combinando estrategias unilaterales de free rider, preeminencia regional y liderazgo (LIMA, 2013, p. 53-60). Sin embargo, más allá de las características comportamentales, la cuestión del "marco temporal" aparece con frecuencia en esta literatura, haciendo que la diferencia entre las potencias medias tradicionales y las emergentes sea crucial para el enfoque comportamental en el capitalismo contemporáneo (COOPER,1997, JORDAAN, 2003, NOLTE, 2007, HUELSZ, 2009).

En consecuencia, Huelsz (2009) identifica seis características de las potencias medias emergentes contemporáneas: (i) tienen una fuerte identidad internacional, que se basa en una visión clara del orden mundial y una comprensión de la posición real y potencial del país dentro de este orden; (ii) son aquellos países que tradicionalmente se encuentran en contextos estructurales diferentes a los de las economías industrializadas, pero cuyas capacidades materiales se han desarrollado en términos que han permitido cierto grado de influencia en la economía global; (iii) su comportamiento tiende a estar influenciado por una agenda global diferente a la de las potencias medias tradicionales; (iv) no necesariamente enfatizan la participación en áreas temáticas que requieren un sentido de responsabilidad ética o moral infundida hacia comunidad internacional; y (v) son aquellos estados cuyas estrategias tienen un carácter "reformador" (vi). Las potencias emergentes también son potencias regionales (HUELSZ, 2009, p.15-16).

En suma, se puede decir que, en el ámbito de la cooperación internacional, las potencias emergentes siguen recibiendo ayudas al desarrollo, aunque ahora también se han comportado como "donantes emergentes", distinguiéndose de los donantes tradicionales por su amplia experiencia como receptores, lo cual les ayuda a comportarse con mayor sensibilidad ante las necesidades y percepciones de los

\footnotetext{
${ }^{7}$ A mediados del siglo $X X$ comenzaron a surgir los primeros debates teóricos sobre potencias medias y emergentes (GIACCAGLIA, 2014), sobre todo en la academia canadiense (HUELSZ, 2009; COOPER y HIGGOTT, 1990; COOPER, HIGGOTT y NOSSAL, 1993) y se desarrollaron aún más a fines de la década de 1990 hasta lo que va del siglo XXI.

8 Las potencias emergentes son típicamente estados grandes con vastos territorios y grandes poblaciones, que a pesar de ser considerados países "en vías de desarrollo", muestran un sorprendente potencial y crecimiento económico.
} 
países menos favorecidos. En este sentido, cabe recordar que el país pionero en esta tendencia como "donante emergente" o "nuevo donante" fue China, que desde principios del siglo XXI amplió las relaciones de cooperación con África y América Latina y el Caribe (MATHIES y STOLTE, 2016, p. 159).

La iniciativa china destaca que "el sistema de ayuda multilateral creado por donantes establecidos parece cada vez más disfuncional” (WOODS 2008, p. 1218), fragmentado y duplicado. Aunque, dialogando con perspectivas opuestas, Mawdsley (2019) argumenta que China fue más prominente y atrajo la atención más temprana, seguida de otros, aunque la geografía de las asociaciones Sur-Sur es muy variada.

Ante ello, Woods (2008) destaca que, paradójicamente, los donantes tradicionales fueron creando tantas instituciones para mejorar la coordinación entre ellos que dejaron de lado al grupo del Banco Mundial que fue un pilar central en el establecimiento del régimen de ayuda para el desarrollo desde la posguerra, en la segunda mitad del siglo XX. En este panorama también es importante recordar que, perversamente, los donantes tradicionales crearon el Banco Mundial pero no dependían de él. En cambio mantenían y expandían sus propias agencias separadas, sobrecargando con un gran número de donantes y diferentes demandas a los gobiernos necesitados de ayuda (WOODS, 2008, p. 1219).

Para tratar de revertir esta distorsión, el Cuarto Foro de Alto Nivel sobre la eficacia de la ayuda, que tuvo lugar en Busan, Corea del Sur en 2011, buscó establecer una fecha para la eliminación de la ayuda vinculada, promoviendo los esfuerzos a nivel mundial y en cada país para aumentar la eficacia de la ayuda como herramienta para reducir la pobreza y alcanzar los Objetivos de Desarrollo del Milenio. Sin embargo, dicho foro se centró en el crecimiento económico para lograr sus objetivos y esto dio lugar a la "tecnificación" de la ayuda, lo cual terminó encajando con la receta de ajuste neoliberal para hacer frente a los efectos de la crisis económica y financiera mundial ${ }^{9}$ (BLYTH, 2013).

Lejos de los debates en los que se enfrascaron los donantes tradicionales, China fue forjado su propia forma de colaboración entre los países del Sur Global, dando nueva vida a la CSS, que, en cierta medida, se había visto debilitada por la lógica del Plan de Acción de Buenos Aires ${ }^{10}$, que al final de la década de 1970

\footnotetext{
${ }^{9}$ Las prácticas neoliberales pueden resumirse en las nuevas formas de mercantilización, privatización y financiarización en un proceso de expansión y profundización de las relaciones capitalistas (OVERBEEK, 2016) que se viene desarrollando desde la década de 1980 (DUMÉNIL y LÉVY, 2014).

${ }^{10}$ El Plan de Acción de Buenos Aires para Promover y Realizar la Cooperación Técnica entre los Países en Desarrollo fue resultado de la Conferencia de Buenos Aires de 1978 que fue acogida por la dictadura cívico-militar de Argentina y por tanto no se puede abstraer del gobierno que la hospedo, es
} 
miraba a la colaboración entre países del Sur como complementaria y, por lo tanto, subordinada a la cooperación Norte-Sur (DOMÍNGUEZ, LO BRUTTO y SURASKY, 2019; DOMíNGUEZ, 2016). Al asumir la existencia o el establecimiento de una relación comercial o de inversión, China, de manera diferente, fue induciendo a los mecanismos de cooperación al distorsionar el significado construido por el Plan de Acción de Buenos Aires ${ }^{11}$.

De esta manera, la presencia de China en la nueva dinámica de la CSS, en el siglo XXI, es una señal importante del desafío a las prácticas neoliberales existentes por parte de las potencias emergentes para conseguir nuevos patrones de desarrollo global (LO BRUTTO y CRIVELLI, 2019, p. 208-209). Todo este proceso ha abierto un debate sobre el ascenso global de China como posible alternativa al orden mundial neoliberal y, por lo tanto, una oportunidad para el Sur Global.

\section{Cooperación Sur-Sur con características chinas}

Existe un amplio consenso académico de que el régimen de cooperación y de ayuda tradicional está atravesando un proceso de transformación. Algunos académicos, como Mawdsley (2012) y Manning (2006) han destacado el importante papel de los "donantes emergentes" y los "donantes que no pertenecen al Comité de Ayuda al Desarrollo (CAD) de la Organización para la Cooperación y el Desarrollo Económico (OCDE) como los principales actores de este proceso. Otros estudiosos, como Lin y Wang (2017a, 2017b) identifican una efectividad cada vez menor de los donantes occidentales tradicionales para facilitar el desarrollo del Sur Global ${ }^{12}$.

decir, el sangriento Proceso de Reorganización Nacional bajo la protección inicial de la doctrina de la Seguridad Nacional de los Estados Unidos. No obstante, con la participación de 138 delegaciones nacionales y más de 2.500 asistentes, la Conferencia de Buenos Aires tuvo se mostró como universal y, por lo mismo, su Plan de Acción sirvió para disciplinar y reconfigurar la Cooperación Económica entre Países en Desarrollo, rediciéndola como Cooperación Técnica entre Países en Desarrollo (DOMINGUEZ, 2019, p. 23).

${ }^{11}$ El Plan de Acción de Buenos Aires restringió los principios de la CSS de Bandung a los objetivos de la cooperación técnica ente países en desarrollo, tratando de promover la autosuficiencia de los países en desarrollo mediante: (a) el fortalecimiento de su capacidad creativa para encontrar soluciones a sus problemas de desarrollo de acuerdo con sus propias aspiraciones, valores y necesidades especiales; (b) la promoción y el fortalecimiento de la autosuficiencia colectiva entre los países en desarrollo mediante el intercambio de experiencias y el uso de sus recursos técnicos; (c) el desarrollo de sus capacidades complementarias; (d) el aumento en la cantidad y calidad de la cooperación internacional; (d) así como la mejora de la eficacia de los recursos para la cooperación técnica mundial mediante el intercambio de capacidades (DE RENZIO Y SEIFERT, 2014).

12 Precisamente desde esta última perspectiva, la definición de AOD de la OCDE, resulta insuficiente ya que ni siquiera incluye algunos de los instrumentos más efectivos para facilitar la transformación estructural en los países receptores, como la inversión de capital y los grandes préstamos no concesionales para infraestructura (LIN y WANG, 2017a). 
Asimismo, Ciertos analistas, como Wood (2008) han interpretado el proceso sistémico de fractura del régimen de la OCDE y del CAD como una "revolución silenciosa en la asistencia al desarrollo" (WOODS 2008). Por su parte, Gore (2013) advierte una situación de "crisis existencial" de la arquitectura de la ayuda institucional, mientras que Domínguez $(2011,2016)$ y Lo Brutto $(2017$; LO BRUTTO y GONZÁLEZ, 2016) destacan que se atraviesa por una "revolución identitaria", como resultado de las contradicciones recurrentes del sistema de ayuda, la proliferación de nuevos actores y disolución de jerarquía Norte-Sur que ordena las relaciones internacionales.

A través del panorama histórico, la Cooperación Sur-Sur se puede reconocer como una expresión de solidaridad entre los países en desarrollo y diversos eventos y procesos políticos globales y regionales que se han proyectado desde la segunda mitad del siglo XX hasta la actualidad generando convergencia de expectativas en torno a principios y motivaciones compartidos. Por eso, cuando se analizan los hechos que intensificaron las prácticas de CSS, se pueden dividir en cuatro períodos según el enfoque de la cooperación en cada momento (MINILLO y ADAD, 2015).

En el primero de estos períodos, es el que va desde el final de la Segunda Guerra Mundial (1945) hasta la Conferencia de Buenos Aires (1978), en el que se establecieron los principios de las relaciones estratégicas entre los países del Sur. En particular, desde la Conferencia de Bandung de 1955 se pueden identificar 46 eventos, en los que se observan las articulaciones políticas de los países del Sur global, en una etapa del proceso de descolonización (LÓPEZ, 2014). Además, esta fase consolidó la cooperación política que dio más protagonismo a los países del Sur en su intento por reducir los desequilibrios del sistema internacional (AYLLÓN, 2015).

La segunda etapa de la CSS comienza después del lanzamiento del Plan de Acción de Buenos Aires (1978) hasta la "Declaración del Milenio" de las Naciones Unidas (2000) en Nueva York ${ }^{13}$. Sin embargo, para Domínguez (2016), la concepción de la CSS delineada en el Plan de Acción de Buenos Aires habría significado una especie de domesticación de la CSS que complementa a la NorteSur existente (DOMÍNGUEZ, 2016, p. 57). No obstante, conviene señalar el proceso de restricción conceptual de la expresión de la CSS en este período, también

${ }^{13}$ Durante el $32^{\circ}$ período de sesiones, la Asamblea General de las Naciones Unidas debatió los principios de la cooperación técnica y la cooperación económica entre países en desarrollo. Después de la Conferencia de Buenos Aires, 138 países adoptaron el Plan el Acción, donde por primera vez se empleó la expresión "cooperación horizontal" como marca registrada de la CSS, contrastando, pero no oponiéndose con la cooperación Norte-Sur "vertical" (UNOSSC, 1978). 
responde a una contradicción entre una nueva etapa de una agenda estrecha de iniciativas de CSS entre los países en desarrollo y una crisis de deuda económica de la década de 1980 que resultó en la llamada "década perdida" en términos de desarrollo en América Latina y el Caribe, iniciada, en particular, por la moratoria mexicana de principios de esa década (LÓPEZ, 2014, MONIZ, 2002) que posteriormente se extendió por toda la región y, en cierta medida, por el resto del mundo también.

La tercera fase inicia en el año 2000 y abarca prácticamente la primera década del siglo XXI, en un periodo en el que, como bien señala Li (2014), la creciente profundización de la globalización económica y el desarrollo constante de las nuevas economías emergentes abrieron la puerta a nuevas oportunidades para la CSS. Esto se debió a que, con la intensificación de la globalización productiva, cada vez más países en desarrollo se fueron incluyendo en la red mundial de fabricación. Esto activó una integración multilateral y regional que resultó en un mercado más abierto para el comercio y la inversión. Además, los nuevos países emergentes brindaron más oportunidades para el crecimiento económico del mundo del Sur en particular, y del mundo en general. Mientras tanto, las crecientes contribuciones internacionales de los países emergentes también permitieron mejorar la infraestructura, el capital humano y el nivel técnico de otros países en desarrollo. Por esas razones, muchos países en desarrollo y subdesarrollados tuvieron mayores expectativas sobre la cooperación Sur-Sur ${ }^{14}$ (Li, 2014, p. 4).

Finalmente, la cuarta fase iniciada en 2009 estuvo marcada por la expansión institucional y por mayor demanda de CSS, sobre todo después de que la crisis de 2008, que puso en tela de juicio el consenso en torno a los principios y reglas de gobernanza del orden económico impulsado por Occidente, y la consiguiente "política de ayuda" gestionada por la OCDE. En este periodo, Li (2016) destaca que con los efectos devastadores de la crisis financiera mundial y el estancamiento de la negociación de la "Ronda de Doha"15 desde 2001, las instituciones como el Banco

\footnotetext{
14 Desde la década de 2000, la CSS ha sido reconocida y promovida en el sistema de cooperación internacional para el desarrollo como complementaria a la cooperación Norte-Sur y hubo convergencia global hacia los objetivos de desarrollo marcados por la agenda de los Objetivos de Desarrollo del Milenio, que estuvieron mayoritariamente enfocados en promover el desarrollo de los países del Sur global (Cabana 2014), siendo especialmente relevante para la CSS el Objetivo 8: "fomentar una alianza mundial para el desarrollo", ya que resalta las necesidades de los países menos favorecidos y propone un esfuerzo generalizado para promover el desarrollo (PNUD, 2020).

${ }^{15} \mathrm{La}$ Ronda de Doha fue la ronda de negociaciones que inició oficialmente en la Cuarta Conferencia Ministerial de la Organización Mundial del Comercio, celebrada en Doha (Qatar) en noviembre de $2001 \mathrm{con}$ el fin de reformar el sistema de comercio internacional mediante el establecimiento de medidas para reducir los obstáculos al comercio y de normas comerciales revisadas (OMC, 2001).
} 
Mundial (BM), el Fondo Monetario Internacional (FMI) y la Organización Mundial de Comercio (OMC), comenzaron a ser cada vez más criticadas y cuestionadas sobre su autoridad discursiva, capacidad de gobernanza y legitimidad institucional. Ello habría desmoronado los pilares de apoyo del orden mundial existente que está experimentando una crisis en su funcionalidad, alcance, legitimidad y autoridad ( $\mathrm{Li}$, 2016, p. 12). En suma, esta fase es una muestra de la metamorfosis de la CSS que cuestiona la eficacia de la asistencia a los países en desarrollo y entre países en desarrollo ${ }^{16}$.

Por lo tanto, es importante mencionar que actualmente la discusión recae en los Objetivos del Desarrollo Sostenible que asignan un rol relevante a la cooperación entre los países del Sur Global. Sin embargo, la discusión sobre la eficacia de la ayuda ha sido, según Lin y Wang (2017), "uno de los temas más complejos y polémicos en la economía del desarrollo en los últimos años, y el debate se ha calentado más recientemente" (LIN y WANG, 2017, p. 54). El argumento actual es que la ayuda tradicional, basada en los principios de la OCDE, es ineficaz para promover el desarrollo y las transformaciones estructurales y ello se sustenta en el hecho de que la cooperación tradicional (Norte-Sur) genera dependencia y cierto nivel de "necesidad de retribución" de las ayudas que reciben, lo cual puede llevar a la corrupción, sobrevaloración y también comprometer el pleno desarrollo de los países receptores al perpetuar la relación de dependencia (KOTHARI y MINOGUE 2001; LIN y WANG, 2017b; RIDDELL, 2008).

Por su parte, China inició su ayuda exterior en 1950 y ella se convirtió en una "cooperación multiforme y de beneficio mutuo" (CHINA-SCPRC 2011, 2), sobre todo a fines de la década de 1970. Cabe señalar que la política de ayuda exterior de China también ha evolucionado a lo largo del tiempo para adaptarse a los cambios de su política exterior, pero siempre siguiendo los Ocho Principios de Ayuda Económica y Asistencia Técnica a Otros Países que fueron establecidos por el Primer Ministro chino Zhou Enlai en 1964. Estos principios pueden resumirse como (i) la igualdad y beneficio mutuo; (ii) la no condicionalidad; (iii) el alivio a la carga de los países receptores; (iv) la autosuficiencia y desarrollo económico independiente; (v) la promoción de proyectos que requieran menos inversión y sean más eficientes; (vi) el equipo de la mejor calidad; (vii) la apropiación por los países beneficiarios de

\footnotetext{
${ }^{16}$ Hasta el año 2013 se realizaron 115 grandes eventos que consolidaron el espacio de CSS en el escenario internacional, así como ampliando el campo de la demanda de diálogo para detectar complementariedades, para formar alianzas destaçando la urgencia de información y mejora de los registros de información sobre esta cooperación (LÓPEZ, 2014).
} 
las técnicas implementadas por China; y (viii) el hecho de que los expertos chinos no pueden disfrutar de servicios especiales (CHINA-SCPRC, 2011, p. 20).

Prácticamente desde su implementación, estos ocho principios se convirtieron en las directrices de la ayuda exterior de la República Popular China, acompañados por otros tres elementos: (i) el internacionalismo, (ii) la búsqueda de la verdad de los hechos, y (iii) y la inclusión de métodos de ayuda y cooperación. Ante ello, queda claro que la cooperación china busca "apoyar a otros países en desarrollo para reducir la pobreza y mejorar los medios de vida de sus pueblos". Además, la cooperación china, pretende ser eficaz al ayudar a "otros países en desarrollo en la construcción de infraestructura, y ayudó a sus esfuerzos en el fortalecimiento de la creación de capacidad y el desarrollo comercial" (CHINA-SCPRC, 2014, 1).

Las observaciones finales de esta sección pretenden subrayar la lenta pero constante tendencia a cambios fundamentales en los principios y procedimientos de implementación de la CSS, recuperando la primera fase, es decir, el espíritu de Bandung, y fusionándolo con el enfoque de "transformación estructural". China está desempeñando un papel de liderazgo en este proceso hacia un nuevo marco de acción y entendimiento que no parece ser replicar la estructura constituida en la esencia de Bandung, ni con el modelo de los países desarrollados sino como una forma china de conducir la CSS.

\section{Cooperación china hacia una transformación estructural}

De acuerdo con Li (2014), la Cooperación Sur-Sur de China con las naciones en desarrollo tiene una dimensión tríadica constituida por el comercio internacional, la inversión y la asistencia exterior. Estas tres "partes focales" constituyen un alcance más amplio de la CSS teniendo en cuenta las relaciones sociales, culturales y políticas. Aunque Li (2014) presenta una definición amplia de CSS, referida a la cooperación entre países en desarrollo, también reconoce que se trata de un concepto evolutivo, que comenzó en las décadas de 1950 y 1960 con la Conferencia de Bandung y el movimiento de Países No Alineados. En este proceso histórico se adaptó no solo con el advenimiento de la globalización y el fortalecimiento de las iniciativas de integración regional en el Sur Global, sino también con el papel destacado de China en la arena internacional.

De hecho, en su Informe de Cooperación Sur-Sur para el Desarrollo, China (2014) define a la CSS, desde su dimensión técnica, como una modalidad 
complementaria a las iniciativas de cooperación Norte-Sur. Sin embargo, Mawdsley (2012) advierte que China reconoce la "ayuda externa" como un componente de la categoría "cooperación para el desarrollo" dividida en: (1) ayuda externa (similar a la asistencia de la OCDE) y (2) apoyo oficial para la cooperación para el desarrollo económico, interconectado con las agendas de comercio e inversiones (MAWDSLEY, 2012, p. 81-82). Esto quiere decir que un atributo destacado de la CSS de China es el énfasis en las asociaciones público-privadas, definidas como una de las formas más inclusivas de cooperación al permitir enfoques basados en el mercado y llenar las brechas en los fondos y técnicas que los recursos públicos disponen (LI, 2013).

En este sentido, China estaría trabajando en los términos definidos por Naciones Unidas, es decir, presuponiendo que la cooperación para el desarrollo SurSur estimula mayores corrientes comerciales y de inversión entre los países en desarrollo contribuyentes y los receptores, es decir, los países menos desarrollados. De esta manera, la CSS sería un catalizador de financiamiento de transacciones de mercado, tales como acuerdos de "recursos naturales para infraestructura", tal y como lo ha hecho China en algunos países africanos en el último cuarto del siglo $X X$. Este estímulo fue levado a cabo por el gigante asiático en la construcción de infraestructura como carreteras, puentes, centrales eléctricas en otros países del Sur a cambio de contratos a largo plazo que garantizan el suministro de materias primas como petróleo, minerales, productos agrícolas, que se traducen en exportaciones hacia territorio chino (UN, 2011, p. 33).

Sin embargo, la estrategia china de cooperación Sur-Sur ha sido criticada por estar orientada a los donantes y no adaptarse adecuadamente a las realidades locales de los países receptores (SHARMA, 2016). A pesar de ello, el discurso oficial de China destaca que sus programas de ayuda se desarrollan en el marco de la cooperación internacional, con sinceridad y sin condicionalidad política. Desde esta perspectiva, las relaciones de cooperación de China con los países emergentes o en desarrollo están respaldadas por flujos comerciales y de inversión anteriores. Esta relación puede formarse a través de acuerdos de cooperación bilaterales, multilaterales o amplios.

En esta dirección, a pesar de que China no impone limitaciones políticas como la democracia liberal, respetando las determinaciones de las instituciones internacionales, el mecanismo económico se convierte en una forma de condicionar la acción de sus socios (MAWSDLEY, 2019; LIN y WANG, 2017a, 2017b). Este 
comportamiento no es restrictivo y, al seguir con su objetivo de ampliar su proceso de inserción global, China opta por establecer relaciones políticas y económicas con sus socios de manera pragmática. No obstante, el tipo de relación construida y la cooperación que surge de la relación establecida tienen relación con los flujos económicos preexistentes o con los acuerdos de cooperación firmados.

En este sentido, China se destaca por su pragmatismo en sus acciones nacionales e internacionales y, según Mawsdley (2019), parece que la complejidad inevitable e inherente de profundizar las relaciones y expandir la inversión significa que los socios más grandes de la CSS pueden tener más dificultades para sostener el reclamo del principio de la 'no interferencia', mientas que sus relaciones e inversiones requieren enfoques más intervencionistas. Por eso, en algunos casos, las infraestructuras institucionales y regulatorias en torno a la CSS se están poniendo al día con esta fase expansiva.

Los tres principales focos de la CSS de China son los países de Asia, África y América Latina y el Caribe, especialmente los países en desarrollo de bajos ingresos. Según la Oficina de Información del Consejo de Estado de China "de 2010 a 2012, China asignó un total de 89.340 millones de yuanes (14.410 millones de dólares) para asistencia exterior en tres tipos: subvención (ayuda gratuita), préstamo sin intereses y préstamo en condiciones favorables" (CHINA-SCPRC, 2014, p. 1) ${ }^{17}$.

El gobierno chino justifica su cooperación diciendo que ayuda a mejorar los "medios de vida de las personas" promoviendo el desarrollo de servicios agrícolas, educativos, médicos y de salud, construyendo instalaciones de bienestar público y ejecutando ayuda humanitaria (CHINA-SCPRC, 2014). En este panorama, por ejemplo, el principal objetivo declarado de la política exterior china para América Latina y el Caribe, como señalaron Harris y Arias (2016), es "aprovechar sus respectivas fortalezas, aprovechar todo el potencial de la cooperación y buscar convertirse en socios mutuamente en la cooperación económica y comercio para beneficio mutuo y desarrollo común" (HARRIS y ARIAS 2016, p. 516).

Todo ello parecía haber quedado claro en los dos Policy Papers que China publicó para los países de América Latina y el Caribe, el primero en 2008 (CHINA, 2008) y el segundo en 2016 (CHINA, 2016), ambos de acuerdo con la definición de CSS aceptada internacionalmente, que implica respeto por la soberanía, no interferencia, mutuo beneficios, entre otros. Sin embargo, a pesar de la retórica de

\footnotetext{
17 Este monto se dividió en acciones para 121 países, 51 de ellos del continente africano (CHINA-
} SCPRC 2014). 
estos principios, Sharma (2016) señala que los proyectos de cooperación chinos, especialmente en el continente africano -pero lo mismo sucedería en los países latinoamericanos y caribeños- han sido objeto de varias controversias por no considerar las características específicas de los países. tales como niveles de justicia, sostenibilidad, inclusión y temas de seguridad como la presencia de grupos terroristas en la región. Tales características pueden ser fatales para la efectividad y eficiencia de los proyectos, especialmente cuando la mayoría de estos proyectos se ejecutan en forma de ayuda financiera (SHARMA, 2016).

La crítica de Sharma a la CSS china está en línea con el discurso de periodistas, académicos y políticos occidentales sobre el papel de China en África. Deborah Brautigam (2015) afirma que la falta de información precisa en la prensa china y los gobiernos africanos aumenta la mayoría de los temores públicos sobre la inversión agrícola china en África en Occidente. En este contexto, las cuatro creencias comunes son: (i) China como un gran comprador de tierras agrícolas con un muchos trabajadores chinos "traídos para trabajar en estas tierras"; (ii) el gobierno chino "lidera el esfuerzo para adquirir tierras en África como parte de un gran plan"; (iii) "Los chinos han desarrollado un apetito voraz por el grano africano" (SOUSA y LEITE, 2018); (iv) "El gobierno chino ha enviado a grandes miembros de campesinos chinos a establecerse en África. Sin embargo, la investigación empírica de Brautigam (2015) muestra que ninguna de estas historias es cierta. De hecho, "según la base de datos de comercio de productos básicos de las Naciones Unidas, es China la que ha estado enviando alimentos a África" (BRAUTIGAM, 2015, p. 3).

Ya en 2012, Dambisa Moyo (2012) refutó la visión convencional sobre el papel de China en África y señaló que la evidencia no respalda la afirmación de que los propios africanos se sientan explotados y, por el contrario, el papel de China parece ser bienvenido en todo el continente. Precisamente, una encuesta del Pew Research Center de 2007 realizada en diez países del África subsahariana encontró que los africanos consideraban abrumadoramente beneficioso el crecimiento económico chino y la participación de China era vista de forma mucho más positiva que la de Estados Unidos ${ }^{18}$ (MOYO, 2012). En efecto, Harris (2015) señala que el fenómeno de la sinofobia podría explicar la incertidumbre que genera la presencia de China en distintas regiones del mundo, provocada por el desconocimiento sobre

\footnotetext{
${ }^{18}$ En este trabajo, destaca que, por ejemplo, en Senegal, el $86 \%$ de los encuestados dieron que el papel de China en su país ayudó a mejorar las cosas, en comparación con el 56 \% que se sentía así sobre el papel de Estados Unidos. En Kenia, el $91 \%$ de los encuestados dijo que creía que la influencia de China era positiva, frente a el $74 \%$ de Estados Unidos (MOYO, 2012).
} 
su cultura y el prejuicio que reflejan algunos ciertos artículos periodísticos y académicos.

Por eso, quizás los mejores exponentes de la posición china contemporánea de CSS son Justin Yifu $\operatorname{Lin}^{19}$ y Yan Wang (2017) que sistematizaron la política de asistencia al desarrollo china, etiquetada como "transformación estructural". Utilizando las palabras de Lin y Wang (2017), la CSS y el desarrollo de China "deberían explicarse más allá de la ayuda" y con ello critican a la denominada cooperación Norte-Sur basada en la Ayuda Oficial al Desarrollo (AOD), que se guía por las directrices de la $O C D E^{20}$, proponiendo en su lugar un enfoque más amplio, como el implementado por China, que combina la ayuda con el comercio y la inversión (LIN Y WANG, 2017a, 2017b).

Esta postura, es, producto de las reflexiones de Justin Yi Fu Lin (2010) en su afán por valorizar el cambio estructural y la modernización industrial que no han recibido la suficiente atención en la literatura económica tradicional, resultando insuficiente a la hora de buscar estrategias efectivas para impulsar un crecimiento sostenible (LIN, 2010, p. ii). Para Lin y Wang (2017a), la cooperación para el desarrollo Sur-Sur utiliza las tres actividades (ayuda, comercio e inversiones) para capitalizar las fortalezas económicas de los países receptores. Esto permite que la CSS para el desarrollo evite los cuellos de botella en los países socios que acostumbran a seguir el modelo estándar de AOD, que separa la ayuda del comercio y la inversión privada, y por lo tanto impide que los países exploten sus ventajas comparativas.

Por tanto, la idea de una Nueva Economía Estructural ${ }^{21}$ trata el desarrollo económico moderno como un proceso de cambio estructural continuo en

\footnotetext{
19 Justin Yifu Lin es uno de los principales assessores del gobierno chino em la elaboración del 14ํㅜ Plan quinquenal de China (2021-2025).

20 La asistencia oficial para el desarrollo (AOD) es definida por el Comité de Asistencia para el Desarrollo (CAD) de la OCDE como ayuda gubernamental que promueve y se dirige específicamente al desarrollo económico y el bienestar de los países. Los flujos de AOD hacia los países y territorios incluidos en la lista de receptores del CAD y hacia las instituciones multilaterales de desarrollo son proporcionado por agencias oficiales, incluidos los gobiernos estatales y locales, o por sus agencias ejecutivas; y en condiciones concesionarias (es decir, donaciones y préstamos en condiciones favorables) y se administra con la promoción del desarrollo económico y el bienestar de los países en desarrollo como objetivo principal (OECD, 2020).

21 Dejando atrás los viejos enfoques de economía estructural basados en sustitución de importaciones que afectaban negativamente a la competitividad de varias cadenas de valor, prohibiendo las importaciones y aumentando los aranceles, la Nueva Economía Estructural optaría más bien por reformular las políticas comerciales con la gradual liberalización del comercio, pero en cuya transición el Estado considere proporcionar cierta protección temporal a las industrias que no son consistentes con la ventaja comparativa del país (LIN, 2012, p. 242).
} 
tecnologías, industrias e infraestructura dura y blanda ${ }^{22}$, todo lo cual aumenta la productividad laboral y, por lo tanto, el ingreso per cápita (LIN Y WANG, 2017a). Según Lin y Wang (2017b), la Cooperación para el desarrollo Sur-Sur es "el intercambio de recursos, tecnología, conocimiento y experiencia entre países en desarrollo, también conocidos como países del Sur Global. Se basa en los principios de solidaridad, respeto mutuo, beneficio mutuo e no interferencia en los asuntos internos" (LIN y WANG 2017b, p. 87).

En efecto, la Nueva Economía Estructural puede ser comprendida como una estrategia de desarrollo acompañada de recomendaciones de política económica (CRIVELLI y LO BRUTTO, 2019, p. 125). De hecho, la metodología del enfoque de transformación estructural explica el desempeño más exitoso de los países en desarrollo comenzando con la observación de que la naturaleza del desarrollo económico moderno es un proceso de cambio estructural continuo en tecnologías, industrias e infraestructura que hace posible el aumento continuo de la productividad laboral y, por lo tanto, el ingreso per cápita en una economía (LIN y WANG, 2017b, p. 22).

En otras palabras, esta perspectiva revalora el papel del Estado en el desarrollo, facilitando la provisión de infraestructura dura y blanda e identificando las ventajas comparativas existentes y latentes del país en un mercado competitivo. Por tanto, el argumento de Lin y Wang (2017) apunta a una dirección en el modus operandi de la cooperación china. La RPC, adoptando su propio modelo de cooperación en el momento actual de la economía global, se basa inicialmente en sus relaciones comerciales y de inversión, y en un segundo momento en el establecimiento de relaciones de cooperación, en particular con países en desarrollo, para establecer una relación de financiamiento mutuo en sectores estratégicos ${ }^{23}$.

En consonancia con esta perspectiva, Li Xing (2017) destaca que el crecimiento de China ha sido precisamente mediante la acumulación y el

\footnotetext{
22 La infraestructura "dura" (hard) es aquello tangible, como las carreteras, puentes, puertos, etc.; mientras que la infraestructura "blanda" o "suave" (soft) es considerado aquello intangible, como el ambiente financiero y legal para la implementación de las inversiones. Ambos tipos de infraestructura son necesarios para facilitar las transacciones de producción y de mercado, permitiendo que la economía llegue a su frontera de posibilidades de producción al reducir los costos de transacción (LIN; WANG, 2017, p. 25).

${ }^{23}$ Es importante dejar en claro que la propuesta de la Nueva Economía Estructural no es equivalente a la política exterior china para el desarrollo del Sur Global. No obstante, se pueden destacar dos importantes puntos de convergencia. En primer lugar, el papel fuerte y activo del Estado para promover el desarrollo y; en segundo lugar, el reconocimiento de que no existe una receta única para lograr el desarrollo.
} 
fortalecimiento de capital a partir de la estrategia de "ocultar el brillo y alimentar la oscuridad" (Tao Guang Yang Hui), y la importancia para ese propósito del paradigma de los "gansos voladores"24 que desde la década de 1960 han guiado el modelo de desarrollo general en Asia Oriental, a partir de un patrón intrarregional de "toma de control", "escalera" y "cadena" de relaciones económicas, mercado y comercio intrarregional e inversión intrarregional” (LI, 2017, p. 2).

El patrón de los gansos voladores describe el orden secuencial del proceso de recuperación de la industrialización de las economías tardías, centrándose en tres dimensiones o etapas: intraindustrial; interindustrial; y división internacional del trabajo. En particular, la última dimensión implica un proceso de reubicación de industrias entre países, desde los avanzados a los en desarrollo, durante el proceso de convergencia de este último grupo. Por lo tanto, una característica que destaca de esta etapa es que las exportaciones de bienes de consumo intensivos en mano de obra disminuyen y los bienes de capital comienzan a exportarse. Dicho de otro modo, en esta etapa, el grupo de economías que siguen el patrón de los gansos voladores avanzan juntas a través de la emulación y el aprendizaje práctico (LIN y WANG, 2017b, p. 30).

Este modelo de cooperación intrarregional se convirtió en una iniciativa global liderada por China desde la Política de "Going Global' lanzada en el siglo XXI por el gobierno de $\mathrm{Hu}$ Jintao $^{25}$ y junto con los Ochos Principios de la ayuda exterior china de Zhou Enlai que han sido plasmados en dos Libros Blancos publicados en 2011 y 2014 respectivamente (CHINA-SCPRC, 2014, p. 2011). Con estos importantes pasos, China perfila nuevas prácticas de ayuda y otras formas de cooperación tratando de mejorar su credibilidad como líder en los asuntos mundiales en un nuevo intento por relanzar el espíritu de Bandung (DE RENZIO y SEIFERT, 2014; AMIN, 2015; 2016).

De esta forma, la región del Asia-Pacífico comienza a emerger como nuevo centro de gravedad mundial y el proyecto de la Ruta de la Seda lanzado por el

\footnotetext{
${ }^{24}$ El paradigma de los gansos voladores fue desarrollado por Kaname Akamatsu (1962) como un modelo para comprender la división internacional del trabajo en el Este de Asia a partir de la ventaja comparativa dinámica, considerando que en una alineación en forma de "V", que imita el patrón de vuelo de gansos salvajes, considera que detrás de un país industrial avanzado se encuentran sucesivamente aquellos países recién industrializados y luego los subdesarrollados hasta llegar a los menos desarrolladas que componen la retaguardia de la formación.

${ }^{25}$ Con un crecimiento económico asombrosamente más alto en las últimas tres décadas y con su creciente acumulación de capital y riqueza, China ha comenzado a aumentar su influencia en los problemas globales y a moldear las agendas y los patrones de gobernanza internacionales. Desde 2010, el mundo ha sido testigo de un patrón desconocido de comportamiento de China en el que se considera que Beijing se ha vuelto cada vez más asertivo e intransigente tanto en el discurso retórico como en la política exterior (LI, 2016, p. 7).
} 
Presidente Xi Jinping en 2013 sustenta la construcción de una nueva arquitectura productiva, una nueva globalización, a partir de una compleja red de interconexión y cooperación en los seis continentes. En este proyecto, cada parte del mundo tiene su lugar $y$, por ende, condiciones de existir en una nueva arquitectura productiva multipolar que implica la inversión y el financiamiento de las obras de infraestructura necesarias para el desarrollo soberano de los bloques nacionales de poder, financiados por herramientas institucionales como el Nuevo Banco de Desarrollo o Banco de los BRICS o el Banco Asiático de Inversiones en Infraestructura (BAII), además de los propios Bancos de Desarrollo de los países donde se asienta la inversión (SHULZ, 2019, p. 39).

La pregunta abierta es si China tomaría el relevo para reconstruir un "nuevo frente en el Sur", es decir, un nuevo Bandung capaz de apoyar las iniciativas independientes de los pueblos y de los Estados del Sur (DOMíNGUEZ, 2019, p. 249). La posibilidad de un nuevo Bandung es muy necesaria para perseguir, de manera coherente y consistente, un resurgimiento del espíritu de solidaridad y cooperación entre los países del Sur para consolidar una alianza hacia el progreso social (ASSI-LUMUMBA, 2015, p. 8). No obstante, quizá todo ello tenga éxito solo en la medida en que China avance en la transformación estructural del Sur Global.

\section{Consideraciones finales}

Si bien China podría competir con el Norte Global en términos de su tamaño, como país en desarrollo, relativamente pobre y humillado por el imperialismo occidental del siglo XIX se define a sí mismo como parte del Sur Global (POLANYILEVITT, 2018, p. 285). De ahí que el estatus de poder de China en la economía mundo capitalista sea complejo, y si bien esta potencia asiática es un centro global en muchas áreas, al mismo tiempo es un país en desarrollo en otras. Con respecto a las relaciones de China con el Sur Global, analizado desde el sistema mundial y las perspectivas de la Economía Política Internacional crítica, China es una potencia central en términos de comercio, inversión y tecnología. La situación es clara si consideramos los vínculos económicos crecientes entre China y Asia oriental y sudoriental, en África y con los países de América Latina y el Caribe.

A la luz de este escenario, Esteves y Assunção (2014) afirmaron que "la coalición del Sur vio el enfoque en la AOD como una reproducción de las asimetrías en la jerarquía política". No obstante, lo contrario es un verdadero dilema ya que la jerarquía política preexistente es la premisa para comprender la reproducción de las 
asimetrías y los intentos de transformismo de la AOD. Por esa razón, en este este artículo se ha destacado que la CSS no tiene una definición única universalmente aceptada y empleada. En la literatura académica que se ha revisado existe una convergencia de expectativas sobre los principios utilizados en este tipo de cooperación si consideramos una perspectiva amplia de la CSS.

El punto de vista chino no separa la ayuda, la inversión, el comercio y la cooperación financiera, lo que implica el respeto por la soberanía, la autodeterminación, la no intervención en los asuntos internos, la ausencia de condiciones y los beneficios mutuos. Estas atribuciones de la CSS se repitieron desde sus inicios en la Guerra Fría con los países del Movimiento No Alineado hasta la actualidad, a través de sus diferentes etapas de desarrollo a pesar de las constantes derrotas contra el régimen hegemónico y condicional de la $A O D$, vinculado al Fondo Monetario Internacional y el Banco Mundial.

De hecho, la definición oficial de CSS del gobierno chino está en línea con tales expectativas (LI, 2012) y el discurso oficial afirma que los proyectos de cooperación chinos se están ejecutando con el objetivo de desarrollo internacional incluso mencionan la agenda de los Objetivos de Desarrollo del Milenio - y beneficios mutuos (CHINA-SCPRC, 2014). Precisamente, Lin y Wang (2017) advierten que la ayuda tradicional para el desarrollo ha sido en gran medida inadecuada para impulsar el crecimiento en las economías de mercado emergentes y, por ello, alcanzar los Objetivos del Desarrollo Sostenible para el 2030, así como combatir el cambio climático según los objetivos en la última reunión del COP21, requerirá grandes cantidades de recursos que parecen inalcanzables (LIN y WANG, 2017, p. 1).

Por eso, no es de sorprenderse que hoy en día, la ayuda, los préstamos y las inversiones chinas en África y América Latina y el Caribe superen con creces a los concedidos por el Banco Mundial y el Fondo Monetario Internacional, convirtiendo a China en el primer y segundo socio comercial de la mayoría de los países del sistema internacional. En este panorama, Vadell y Ramos (2015) destacan que, desde la crisis financiera de 2008, es posible percibir un déficit creciente en la inversión en infraestructura, especialmente en el mundo en desarrollo. Según una investigación de la OCDE, para 2030 se necesitarían aproximadamente 70 billones de dólares para atender las demandas de inversión en electricidad, agua, transporte de telecomunicaciones y generación, transmisión y distribución de energía (OCDE, 2006, 2007). Además, la inversión privada disminuyó desde la crisis de 2008 
(WIGGLESWORTH, 2012), los bancos multilaterales de desarrollo y la AOD limitaron las inversiones en proyectos de infraestructura en menos del 10\% para los países menos desarrollados y para las potencias medianas emergentes (CHIN, 2014).

La función y responsabilidades como inductor del desarrollo las estaba cumpliendo China a través de la estrategia que captura la esencia de los principios de CSS de Bandung y Zhou Enlai con la perspectiva amplia de la cooperación al desarrollo, integrando la tríade: ayuda, comercio e inversiones en un solo principio. Este enfoque no restrictivo de la CSS llega en un momento oportuno para que los países menos desarrollados provoquen cambios en el régimen internacional de ayuda y cooperación. El concepto denominado "cooperación al desarrollo para la transformación estructural", desarrollado por Ling y Wang (LIN y WANG, 2017b), está en consonancia con el enfoque del gobierno chino.

Por eso no es de sorprenderse, que entre los nueve expertos convocados por el presidente Xi Jinping para delinear el Decimocuarto Plan Quinquenal, 2021-2025, se encuentre Justin Yifu Lin, que es un defensor de que el Estado asuma un papel activo en la planificación industrial para ayudar a los países en desarrollo a ponerse al día rápidamente con las economías avanzadas (TANG, 2020). De hecho, el Libro Blanco sobre el Desarrollo Pacífico, China (2014) defiende las instituciones multilaterales para promover el desarrollo y la paz, al mismo tiempo que promueve nuevas alianzas regionales estratégicas con el Sur Global, basadas en una política exterior china más asertiva bajo el liderazgo de Xi Jinping. (LI, 2017). Esto encaja en el debate sobre el enfoque de una nueva gran estrategia china de "lucha por el logro" (You Suo Zuo Wei) (QIN, 2014; YAN, 2014) que representaría "un cambio hacia una política exterior más proactiva frente a los nuevos desafíos y limitaciones del mundo actual liderado por Estados Unidos" (LI, 2016, p. 21).

Según Domínguez (2018), la CSS es parte esencial de la identidad china porque los aportes fundamentales de este país - comenzando por los cinco principios de convivencia pacífica de Zhou Enlai que sistematizaron el llamado espíritu de Bandung - aún son uno de los pilares de la doctrina oficial de la cooperación china y, por lo tanto, de su política externa. En este sentido, la idea de un nuevo espíritu de Bandung crea un nuevo desafío para el Sur Global: qué puede hacer el Sur por sí mismo mediante la cooperación Sur-Sur y cómo estos países pueden influir en el orden mundial a través de la acción colectiva (DOMÍNGUEZ, 2018, p. 53). 
Con base en lo expuesto en este artículo, se concluye que el enfoque de transformación estructural del desarrollo está creando reglas desafiantes para el régimen de CSS occidental en el sistema capitalista mundial. Además, cabe decir que desde el estallido de la pandemia del Covid-19, China también se ha puesto a la vanguardia de la cooperación sanitaria, lo que se presenta como una faceta complementaria de su diplomacia económica y liderazgo global (RUBIOLO y VADELL, 2020). En este contexto, las nuevas dinámicas de cooperación de China se han ido alejando de los esquemas de ayuda tradicionales, cubriendo la CSS con nuevas prácticas de diálogo político, acuerdos comerciales y financiamiento de infraestructura, lo que abre las puertas a un posible nuevo régimen de cooperación, una especie de Bandung 2.0.

\section{REFERÊNCIAS}

AKAMATSU, K. (1962):"A historical pattern of economic growth in developing countries". Journal of Developing Economies, V. 1, N. 1, p. 3-25.

AMIN, S. "From Bandung (1955) to 2015: Old and New Challenges for the States, the Nations and the Peoples of Asia, Africa and Latin America". International Critical Thought, V. 5 N. 4, p. 453-460. 2015.

ASSIE-LUMUMBA, N'D. T. "Behind and beyond Bandung: historical and forward-looking reflections on south-south cooperation. Bandung: Journal of the Global South, V.2, N.11, p. 1-10, 2015.

ASIAN-AFRICAN-CONFERENCE. Final Communiqué of the Asian-African conference of Bandung. 1955.

AYLLÓN, B. "La conferencia Afroasiática de Bandung: hito de las relaciones Sur-Sur". Boletim Mundorama. 2015.

BERGAMASCHI, I. Y DURAN, J. "Conclusion: South-South Cooperation Experiences Compared and the Way Forward". In BERGAMASCHI, I., MOORE, P. y TICKNER, A. B. (eds.). South-South Cooperation Beyond the Myths. London: Palgrave Macmillan, 2017, p 301-324.

BLYTH, M. Austerity: the history of a dangerous idea. Oxford: Oxford University Press. 2013.

BRAUTIGAM, D. Will Africa Feed China? New York: Oxford University Press. 2015.

CHIN, G. T. "The BRICS-led Development Bank: Purpose and Politics beyond the G20." Global Policy, V.5, N.3, p. 366-373. 2014.

CHINA. China's Policy Paper on Latin America and the Caribbean. State Council The People's Republic of China. 2008.

CHINA. China's Policy Paper on Latin America and the Caribbean. State Council The People's Republic of China. 2016. 
CHINA-SCPRC. White Paper China's Foreign Aid. State Council The People's Republic of China. 2011.

CHINA-SCPRC. White Paper China's Foreign Aid. State Council The People's Republic of China . 2014.

CRIVELLI, E. y LO BRUTTO G."La cooperación de China en América Latina: ¿hacia una Nueva Economía Estructural?" Carta Internacional, V. 13. N. 2. p. 123-146. 2018.

COOPER, A. F. Niche diplomacy: middle powers after the Cold War, Studies in diplomacy. New York: Macmillan. 1997.

COOPER, A. F. y HIGGOTT, R. A. "Middle power leadership and coalition building : the Cairns Group and the Uruguay Round, March 1990", Working paper. Canberra: Research School of Pacific Studies, Australian National University. 1990.

COOPER, A. F., HIGGOTT, R. A., y NOSSAL, K. R. Relocating middle powers : Australia and Canada in a changing world order, Canada and international relations. Vancouver: UBC Press. 1993.

DE RENZIO, P. y SEIFERT, J. "South-South cooperation and the future of development assistance: mapping actors and options". Third World Quarterly, N. 35, V.10, p. 1860-1875, 2014.

DOMíNGUEZ, R. "La Crisis de Identidad del sistema de ayuda". Fundación Carolina. 2011.

DOMíNGUEZ, R. "En los pliegues de la historia: Cooperación Sur-Sur y procesos de integración en América Latina y el Caribe" Estudos Internacionais, V. 4, N. 2, p. 57-77. 2016.

DOMÍNGUEZ, R. "China y la construcción de un régimen internacional de Cooperación SurSur". Carta Internacional, V. 13, N. 1, p. 38-72, 2018.

DOMÍNGUEZ, R. "La constelación del Sur: la cooperación Sur-Sur, en el cuarenta aniversario del Plan de Acción de Buenos Aires". In DOMíNGUEZ, R. , LO BRUTTO, G. y SURASKY, J. (ed.) (2019). La Constelacion del Sur, lecturas Historico-Criticas de la Cooperacion Sur-Sur. Mexico: BUAP-UC, 2019, p. 13-131.

DOMÍNGUEZ, R. , LO BRUTTO, G. y SURASKY, J. (ed.) (2019). La Constelacion del Sur, lecturas Historico-Criticas de la Cooperacion Sur-Sur. Mexico: BUAP-UC, 2019.

DUMÉNIL, G. y LÉVY, D. A Crise do Neoliberalismo. São Paulo: Boitempo.2014.

ESTEVES, P. y ASSUNÇÃO, M. "South-South Cooperation and the International development battlefield: between the OECD and the UN." Third World Quarterly, V. 35, N. 10, p. 775-1790, 2014.

GIACCAGLIA, C. "Brasil desde la mirada argentina. El activismo internacional brasileño en los ámbitos multilaterales" In BERNAL, R. y BIZZOZERO L. (eds.) La política internacional de Brasil: de la región al mundo. Montevideo: Cruz del Sur. 2014. p. 79-100.

GORE, C. "The New Development Lanscape: Actors, Approaches, Architecture". Journal of International Development, N. 25, p. 769-786, 2013.

HARRIS, R. L. "China's Relations with the Latin American and Carribean Countries: A

Peaceful Panda Bear instead of a Roaring Dragon." Latin American Perspectives, V. 42,

N. 6, p. 153-190. 2015. 
HARRIS, R L., y ARIAS, A. A. "China's South-South Cooperation with Latin America and the Caribbean." Journal of Developing Societies, V. 32, N. 4, p. 508-556, 2016.

HUELSZ, C. "Middle Power Theories and Emerging Powers in International Political Economy: A Case Study of Brazil." PhD Thesis University of Manchester, 2009.

JORDAAN, E."The concept of a middle power in international relations: distinguishing between emerging and traditional middle power." Politikon, V. 30, N. 2, p. 165-18, 2003.

KOTHARI, U. y MINOGUE, M. Development Theory and Practice: Critical Perspectives. Basingstoke: Palgrave, 2001.

LEITE, A. C. C. "O Atual Momento do Desenvolvimento Chinês: planejamento regional, investimento e comércio internacional." In VADELL, J. A. Expansão Econômica e Geopolítica da China, Belo Horizonte: PUC Minas, 2018, p. 259-283.

LI, R. "China Development Report on South-South Cooperation" ecdc.net, 2013. http://www.ecdc.net.cn/doc/sscreport.pdf . 2013. Acceso 15. oct. 2017.

LI, X."The Expansion of China's Global Hegemonic Strategy: Implications for Latin America." Journal of China and International Relations V. 4 (Special Issue), p. 1-26, 2016.

LI, X. "The Rise of Emerging Powers \& China and the Enlargement of 'Room for Maneuver' and 'Upward Mobility'. Rising Powers in Global Governance, 2017.

LI, X. "Understanding the Multiple Facets of China's "One Belt One Road" Initiative." In LI, X. (ed.), Mapping China's 'One Belt One Road' Initiative: London/New York: Palgrave Macmillan, 2019.

LIMA, M. R. S. de. The Political Economy of Brazilian Foreign Policy: Nuclear Energy, Trade and Itaipu. Brasília: FUNAG, 2013.

LIN, J. Y. Nueva Economía Estructural, un marco para reformular el desarrollo. Documento de trabajo sobre investigación de políticas del Banco Mundial, N. 5197, p. 1-39, 2010.

LIN, J. Y. New Structural Economics, A Framework for Rethinking Development and Policy. Washington D.C.: World Bank, 2012.

LIN, J. Y. y WANG. Y. Development Beyond Aid. Project Syndicate. 2017a.

LIN, J. Y. y WANG. Y. Going Beyond Aid. Cambridge: Cambridge University Press, 2017b.

LO BRUTTO, G. "A Propósito de la Cooperación Internacional y del Desarrollo." In DOMÍNGUEZ R. y RODRIGUEZ G. (coords.) Historia de la Cooperación Internacional desde uma perepectiva crítica. Barranquilla: Uniautónoma, 2017, p. 57-79.

LO BRUTTO, G. y GONZÁLEZ C. H."Realidades y desafíos contemporáneos de la cooperación sur-sur en América Latina y el Caribe." Estudos Internacionais, V.4, N. 2, p. 79-92, 2016.

LO BRUTTO, G. y CRIVELLI, E. "Las relaciones de China con América Latina frente al cambio político latinoamericano." In DOMíNGUEZ, R., LO BRUTTO, G. y SURASKY, J. (coords.). La Constelación del Sur: lectiuras histórico críticas de la cooperación SurSur. México: BUAP-UC. 2019, p. 207-238.

LOPEZ, S. "Chronology and history of south-south cooperation: An IberoAmerican Contribution". Working Document Mastergraf, N. 5, 2014. 
MANNING, R. "Will 'emerging Donors'Change the Face of international Co-operation?" Development Policy Review, V. 24, N. 4, p. 371-385, 2006.

MAWDSLEY, E. From recipients to donors: emerging powers and the changing development landscape. London/New York: Zed Books, 2012.

MINILLO, X. K. P. y ADAD, B. "O Regime Internacional de Cooperação Sul-Sul: Invisibilização Institucional e Teórica." 5 Encontro Nacional Associação Brasileira de Relações Internacionais, 2015.

MONIZ, L. A. "As políticas neoliberais e a crise na América do Sul" Revista Brasileira de Política Internacional, V.45, N. 2, p. 35-146, 2002.

MORVARIDI, B. y HUGHES, C. "South-South Cooperation and Neoliberal Hegemony in a Post-aid World." Development and Change, V. 49, N. 3, p. 867-892, 2018.

MOYO, D. "Beijing, a Boom for Africa." New York Times, 27 jun. 2012. http://www.nytimes.com/2012/06/28/opinion/beijing-a-boon-for-africa.html. Acceso 7 nov. 2017.

NOLTE, D. "How to Compare Regional Powers: Analytical Concepts and Research Topics." ECPR Joint Session Workshops, 2007.

OECD. "Official Development Assistance (ODA), What is ODA?". oecd.org. abr. 2020. http://www.oecd.org/dac/financing-sustainable-development/development-financestandards/What-is-ODA.pdf. Aceso 25 sep. 2020.

OECD. Infrastructure to 2030: Telecom, Land Transport, Water and Electricity. OCDE Publishing, 2006.

OECD. Infrastructure to 2030 (Vol. 2): Mapping Policy for Electricity, Water and Transport. OCDE Publishing, 2007.

OMC. "Declaración Ministerial". Doah: Ministerial de la OMC. 14 nov. 2001. https://www.wto.org/spanish/thewto_s/minist_s/min01_s/mindecl_s.htm Acceso 25 sep. 2020.

OVERBEEK, H. Globalizing China: A Critical Political Economy Perspective on China's Rise." In CAFRUNY. A., L. S. TALANI y POZO G. The Palgrave Handbook of Critical International Political Economy. London: Palgrave Macmillan, 2016, p. 309-329.

PNUD. Objetivos del desarrollo del milenio. undps.org. 2020. https://www.undp.org/content/undp/es/home/sdgoverview/mdg_goals.html. Acceso 25 sep. 2020.

POLANYI-LEVITT, K. De la gran transformación a la gran financiarización, sobre Karl Polanyi y otros ensayos. México: FCE.

QIN, Y. "Continuity through Change: Background Knowledge and China's International Strategy." The Chinese Journal of International Politics, V.7, N. 3. p. 285-314, 2014.

RUBIOLO, F. y VADELL, J. China, América Latina y la 'diplomacia de las mascarillas'. EI País, 29 jun. 2020. http://agendapublica.elpais.com/china-america-latina-y-la-diplomacia-delas-mascarillas/. Acceso 25 sep. 2020. 
SCHULZ, J. S. La Nueva Ruta de la Seda. Universalismo y pluriversalismo para un nuevo orden mundial. Análisis y pensamiento Iberoamericano sobre China. N. 32, p. 24-46. 2019.

SHARMA, P. Evolving Nature of China's South-South Cooperation. Global Issues, 2016.

SHAW, T. M., COOPER, A. F. y ANTKIEWICZ, A. "Global and/or Regional Development at the Start of the 21 Century? China, India and (South) Africa." Third World Quarterly, V. 28, N. 7, p. 1255-1270, 2007.

SOUSA, M. E. de A. y LEITE, A. C. C. "O Dinamismo das Aquisições Transnacionais de Terra: aproximando o fenômeno das Relações Internacionais e da Economia Política Internacional." Estudos Internacionais, V. 5, N. 2, p. 13-33, 2018.

TANG, F. "China's economic future is being influenced by nine economists, but what did they tell Xi Jinping this week?" South China Morning Post.

https://www.scmp.com/economy/china-economy/article/3099294/chinas-economic-future-

being-influenced-nine-economists-what. Acceso, 25 sep. 2020.

\section{UN. Fourth United Nations Conference on the Least Development Countries, Harnessing the Positive Contribution of South-South Co-operation for Least Developed Countries' Development. UNOSSC, 2011.}

UNOSSC. Buenos Aires Plan of Action. New York: UN, 1978.

VADELL J. y RAMOS, L. "As sedutoras garras do dragão chinês: Finanças e Bifurcação do Centro da Economia Global". Grupo de Pesquisa sobre Potências Médias, 05 abr. 2015. https://grupoemergentes. wordpress.com/2015/04/05/as-sedutoras-garras-do-dragao-chinesfinancas-e-bifurcacao-do-centro-da-economia-global/. Acceso. 20 mar. 2016.

VADELL, J., RAMOS, L. y NEVES, P. "The international implications of the Chinese model of development in the Global South: Asian Consensus as a network power" Revista Brasileira de Política Internacional, V. 57 (special issue), p. 91-107, 2014.

WIGGLESWORTH, R. "Infrastructure projects face funding gap". Financial Times, 2012.

WOODS, N. "Whose aid? Whose influence? China, emerging donors and the silent revolution in development assistance". International Affairs, V. 84, N. 6, p. 1205-1221, 2008.

YAN, X. "From Keeping a Low Profile to Striving for Achievement" The Chinese Journal of International Politics, V. 7, N. 2, p. 153-184, 2014.

ZHANG, Q. China's relations with developing countries: patterns, principles, characteristics, and future challenges. In FREEMAN, C. Handbook of Research on Contemporary China. London: Edward Elgar, 2016, p. 51-70. 
CONTRIBUIÇÃO DE AUTORIA

\section{NOTAS DE AUTOR}

Javier Alberto Vadell - Concepção. Coleta de dados, Análise de dados, Elaboração do manuscrito, revisão e aprovação da versão final do trabalho

Giuseppe Lo Brutto - Concepção e elaboração do manuscrito. Coleta de dados Participação ativa da discussão dos resultados; Revisão e aprovação da versão final do trabalho.

Alexandre Cesar Cunha - Concepção. Coleta de dados, Análise de dados, Elaboração do manuscrito, revisão e aprovação da versão final do trabalho

Eduardo Crivelli - Concepção e elaboração do manuscrito. Coleta de dados Participação ativa da discussão dos resultados; Revisão e aprovação da versão final do trabalho.

\section{FINANCIAMENTO}

Não se aplica.

\section{CONSENTIMENTO DE USO DE IMAGEM}

Não se aplica.

\section{APROVAÇÃO DE COMITÊ DE ÉTICA EM PESQUISA}

Não se aplica.

\section{CONFLITO DE INTERESSES}

Os autores declaram não haver conflito de interesses.

\section{LICENÇA DE USO}

Este artigo está licenciado sob a Licença Creative Commons CC-BY. Com essa licença você pode compartilhar, adaptar, criar para qualquer fim, desde que atribua a autoria da obra.

\section{HISTÓRICO}

Recebido em: 29-09-2020

Aprovado em: 23-10-2020 